\title{
Effectiveness of visible and ultraviolet light emitting diodes for inactivation of Staphylococcus aureus, Pseudomonas aeruginosa, and Escherichia coli: A comparative study
}

\author{
Sameen Ahmed Malik ${ }^{a}$, Tan Tian Swee ${ }^{a *}$, Nik Ahmad Nizam Nik Malek ${ }^{a}$, Azli Yahya ${ }^{b}$, Takahiro \\ Emoto c, Masatake Akutagawa c, Leong Kah Meng a, Tan Jia Hou a, Tengku Ahmad Iskandar \\ Tengku Alang a, Kelvin Ling Chia Hiik ${ }^{a}$ \\ a Department of Biotechnology and Medical Engineering, Faculty of Biosciences and Medical Engineering, Universiti Teknologi Malaysia, Skudai \\ 81310, Johor, Malaysia \\ ${ }^{b}$ Department of Clinical Sciences, Faculty of Biosciences and Medical Engineering, Universiti Teknologi Malaysia, 81310 Skudai, Malaysia \\ c Department of Electrical and Electronic Engineering, Institute of Technology and Science, The University of Tokushima, 2-1 Minamijyosanjima- \\ cho, Tokushima 770-8506, Japan \\ * Corresponding author: tantswee@biomedical.utm.my
}

\section{Article history}

Received 30 June 2018

Revised 23 September 2018

Accepted 23 October 2018

Published Online 25 August 2019

\begin{abstract}
The rapid use of ultraviolet light emitting diodes (UV-LEDs) in various disinfection applications is growing tremendously due to their advantages unachievable using UV lamps. In this study, a comparison of standard LED at $460 \mathrm{~nm}$ wavelength and UVA LED at $385 \mathrm{~nm}$ was conducted to determine their effectiveness in disinfection of frequently isolated pathogens in hospitals (Staphylococcus aureus, Pseudomonas aeruginosa, and Escherichia coli). Determination of disinfection efficiency was carried out by measuring inhibition zone. Effects of varied exposure time on the inactivation of pathogenic microorganisms was studied. The results demonstrated that LED does not have germicidal activities. The highest inactivation for UVA LED was achieved for Pseudomonas aeruginosa. Linear relationship was found between exposure time and log reduction. This study showed that UVA LEDs can effectively inactivate significantly higher number of microorganisms hence can be used in disinfection of various applications.
\end{abstract}

Keywords: Visible LED, UVA-LED, pathogens, inactivation effectiveness

\section{INTRODUCTION}

Infections that patients acquire while having treatment in the healthcare are known as healthcare associated infection (HAIs). HAIs are considered to be one the most leading causes of illnesses and deaths worldwide. HAIs can occur in hospitals, care homes, and even in patient's own house (Van Kleef, Robotham, Jit, Deeny, \& Edmunds, 2013). It is reported that in United States alone around 1.7 million people contacted HAIs each year and causing deaths among 99,000 patients (Brannigan \& Holmes, 2012). Cruickshank and Ferguson estimated that as many as 200,000 HAIs cases are recorded in Australia annually which makes it the most common complication effecting patients in hospitals (Cruickshank, Murphy, \& eds., 2009). Some of the most frequent microorganisms, responsible from HAIs, are the Staphylococcus aureus, Escherichia coli, and Pseudomonas aeruginosa (Donlan, 2001). They are known to cause infections such as pneumonia, respiratory tract infections, urinary tract infections, and surgical site infections (Al-Tawfiq \& Tambyah, 2014).

Medical device associated infections also pose significant risk to the patients and medical personnel in healthcare that resulted in increases morbidity and mortality (Donlan, 2008). Routine disinfection of non-critical medical devices such as stethoscopes, blood glucose meters, and blood pressure cuffs are important to protect vulnerable patients from nosocomial infections. It has been reported that noncritical medical devices rarely get disinfected between uses with different patients which increases the likelihood of infecting any patient getting in contact with these devices (Bukharie, Al-Zahrani, Rubaish, \& Abdulmohsen, 2004; Dancer, 2012; Uneke et al., 2014; Uneke, Ogbonna A Fau - Oyibo, Oyibo Pg Fau - Ekuma, \& Ekuma, 2008; Zachary et al., 2001). In fact, in some studies the percentage of contamination on these devices could range between $25 \%$ to $100 \%$ (Bukharie et al., 2004; Chigozie Jesse, 2014; D, S, R, G, \& S, 2016; Grewal, Varshney, Thomas, Kok, \& Shetty, 2013).

Existing disinfection and sterilization practices in healthcare such as the use of chemicals, dry heat, and steam have significant limitations which make medical personnel to neglect the disinfection protocols. Some of the limitations are as follows: i) long and tedious procedures; ii) cause skin irritation and respiratory diseases; iii) alter surface structure of the medical device. Alternative methods such as ultraviolet (UV) light can help to overcome existing limitations thereby increasing the quality of human life. The importance of UV irradiation in everyday life is ever increasing with its ability in various applications ranging from disinfection to tanning and food preserving and many more. UV light is already replacing traditional disinfection practices in water treatment and same promising future can be predicted in the healthcare.

The use of mercury based UV lamps is very common when it comes to modern disinfection technologies. UV mercury based lamps are widely used in disinfection of water and now the use of these lamps in healthcare is also increasing. These lamps make use of mercury vapor to produce UV light and can be categorized into two main types: 
monochromatic and polychromatic. Monochromatic are referred to as low-pressure (LP) mercury lamps and are a type of monochromatic which emit most of UV light at $253.7 \mathrm{~nm}$ wavelength. Whereas polychromatic which is also known as medium pressure (MP) can produce UV light at various wavelengths (Kowalski, 2009; Linden, Thurston, Schaefer, \& Malley, 2007). Tru-D is one of many commercial products making use of mercury vapor principle to produce light. Numerous studies have been carried out to study the effectiveness of these devices in healthcare. The results indicated that Tru-D can efficiently inactivate significant amount to pathogens from healthcare surfaces and devices (Anderson et al., 2013; Mahida, Vaughan, \& Boswell, 2013; Nerandzic, Cadnum, Pultz, \& Donskey, 2010).

Unfortunately, these lamps pose many drawbacks which makes their use in the modern era highly unfavorable. The lamps are generally made of fragile quartz material hence the risk of mercury leakage is always present throughout the lifecycle of the lamps (Shin, Kim, Kim, \& Kang, 2016). The lamps require warm-up time before operation therefore instantaneous disinfection cannot be carried out. Moreover, they require high voltage and produce a lot of heat during operation (Yoshihiko, Masahiro, \& Suguru, 2014). The frequent replacement of these lamps is very common due to extremely short lifecycle (Hölz, Lietard, \& Somoza, 2017). Additionally, it can only be used in continuous mode and thus, have to be remained switched on throughout entire disinfection process. Aforesaid limitations do not allow these lamps to be used in point-of-care (POC) disinfection applications (Chen, Loeb, \& Kim, 2017).

One of the most feasible alternatives to UV lamps are the UV light emitting diodes (UVLEDs). The use of UVLEDs is on the rise due to latest technical advancements in this technology. Furthermore, UVLEDs offer benefits that seem impractical using conventional lamps. These environmentally-friendly lamps do not make use of mercury contents and produce ozone-free UV light (Eskandarian, Choi, Fazli, \& Rasoulifard, 2016). Highly compact size allows them to be used in portable and POC applications. Almost all the energy is converted into UV light and only small amount of energy is wasted as heat (McDermott, Walsh, \& Howard, 2008; Vilhunen, Särkkä, \& Sillanpää, 2009). These LEDs are available in UVC, UVB, and UVA regions and the wavelengths can be selected based on the type of microorganisms to be disinfected. Extremely long lifetime, low operation voltage, and no warm-up time required are some of many advantages of UVLEDs (Chatterley \& Linden, 2010; Matafonova \& Batoev, 2018; Messina et al., 2016).

The efficiency of UVLEDs in disinfection of water (Chatterley \& Linden, 2010; Matafonova \& Batoev, 2018; Oguma, Kita, \& Takizawa, 2016), food (Kim, Kim, \& Kang, 2016; Shin et al., 2016; Shirai, Watanabe, \& Matsuki, 2017), and healthcare (Donlan, 2008; G. Messina, Burgassi, Messina, Montagnani, \& Cevenini, 2015; Gabriele Messina et al., 2016; Omotani et al., 2018) has been reported by numerous studies which suggest that UVLEDs can be effectively used for said applications. The UVLEDs are already in process of replacing traditional UV lamps (Yoshihiko et al., 2014). Comparative studies between UVLEDs and UV lamps have also been carried out to determine the most effective technology in disinfection. The results concluded that in some cases UVLEDs are as effective as UV lamps (Beck et al., 2017; Sholtes et al., 2016) while in other they are even more effective (Li, Wang, Huo, Lu, \& Hu, 2017).

Majority of research has been directed towards UVC-LEDs for disinfection applications and comparatively limited research can be found on UVA-LEDs. It is well established that UVC-LEDs have higher inactivation efficiency than UVA, however, both have disinfection properties. Unfortunately, damaged caused by UVC can be easily repaired using photoreactivation process whereas UVA can withstand photoreactivation hence making UVA long-lasting disinfection as compared to UVC. Furthermore, UVA-LEDs are much more energy efficient, have higher optical output power, and are far cheaper than UVC (Aoyagi et al., 2011; Harris, Pagan, \& Batoni, 2013; Yoshihiko et al., 2014). In this study, inactivation efficiency of a standard visible light LED, and UV LED has been compared for disinfection of Staphylococcus aureus, Pseudomonas aeruginosa, and Escherichia coli.

\section{EXPERIMENTAL}

\section{Experimental design}

A standard visible LED (F33CC4SB-3) at $460 \mathrm{~nm}$ wavelength was used in conjunction with UVA LED to compare inactivation efficiency of both light sources. UVA LED at $385 \mathrm{~nm}$ (NVSU233A(T)-D1) was purchased from Nichia, Japan. The following factors were kept into consideration for both light sources: i) light source must have significantly higher output optical intensity; ii) LED must be compact in size as to fit in portable applications; iii) requires low DC voltage for operation. The Emission spectrum of LED and UVA LED is shown in Fig. 1 (A) and (B) respectively. DC power supply was used to operate the LEDs. In order to operate LEDs efficiently, voltage regulator and current limiter circuits were designed. Due to input voltage and current difference between both sources, LED was powered on after stepping down the voltage and current while UVA LED was directly switched on from the source. LED was applied with $30 \mathrm{~mA}$ current while UVA LED was powered on with $700 \mathrm{~mA}$. The distance between the source and the sample, for both light sources, was kept at $7 \mathrm{~cm}$ in order to ensure even distribution of light across the petri dish as illustrated in Fig. 2.
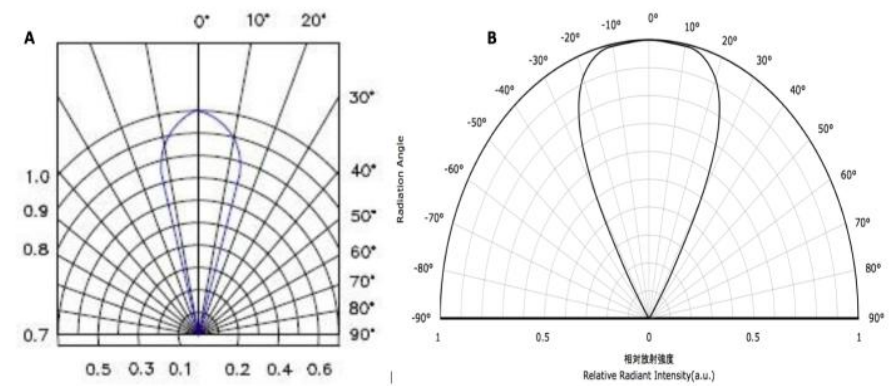

Fig. 1 Emission spectrum A) standard LED B) UVA.

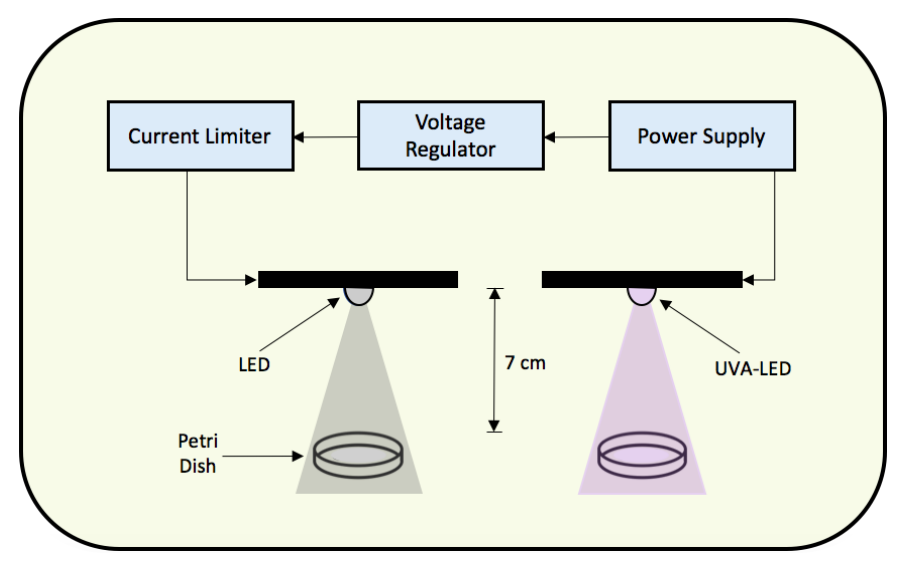

Fig. 2 Schematic representation of experimental setup.

\section{Microorganisms preparation}

Microorganisms that cause serious illness and are frequently isolated from healthcare (Khurram, Umar M Fau - Akhter, Akhter, Hamam-Tul-Bushra Fau - Faheem, \& Faheem, 2013; Sserwadda et al., 2018) were selected for this research study. Staphylococcus aureus (ATCC 6538), Pseudomonas aeruginosa (ATCC 15442), and Escherichia coli (ATCC 11229) were used to determine the inactivation effectiveness of LED and UVA LED. In order to obtain single colony, all bacteria were first streaked on nutrient agar plate using sterilized inoculation loop. After incubation at $37^{\circ} \mathrm{C}$ for $24 \mathrm{~h}$, the bacterial colonies were observed before further use. Approximately 5 to 7 bacterial colonies of $S$. aureus were added to $1 \mathrm{ml}$ of saline solution using inoculation loop and turbidity of the solution was compared with $0.5 \mathrm{McF}$ arland to achieve desired concentration of $1.5 \times 10^{8} \mathrm{CFU} / \mathrm{ml}$. The bacteria solution was swabbed onto nutrient agar dish using cotton bud and left to dry before sealing it with parafilm. The petri dishes of all bacteria were prepared for LED and UVA LED samples. 


\section{Exposure to light source}

For each experiment, a set of three petri dishes were used: for control, LED, and UVA LED samples. Each petri dish was kept in a separate box with light source on top, facing downwards. Both light sources had similar source/sample distance and were operated in continuous wave $(\mathrm{CW})$ throughout the experimental period to investigate their effects on the bacteria. All experiments were conducted in well ventilated and sterilized environment as to reduce contamination. All boxes including control were covered with lids to avoid outer light influence on the samples. Exposure time was varied while constantly exposing the petri dishes to their respective lights. Different exposure times $(1,5,15,30$, and $60 \mathrm{~min})$ were examined for all three microorganisms. The UV dose induced to the sample after $1 \mathrm{~h}$ of continuous exposure was $57.6 \mathrm{~mJ} / \mathrm{cm}^{2}$. After treatment, the petri dishes were sealed with parafilm and incubated at $37^{\circ} \mathrm{C}$ for approximately $24 \mathrm{~h}$ to observe the bacteria growth.

\section{Determination of inhibition zones}

To determine antibacterial efficiency of LED and UVA LED sources on the bacteria, zone of inhibition test was conducted. As the diameter of the zone directly corresponds to the sensitivity of light source on the microbes, therefore higher inhibition zone would correlate with high inactivation efficiency and vice versa. The zone of inhibition was measured and recorded in millimeter unit.

\section{RESULTS AND DISCUSSION}

\section{Inactivation effects of LED on microorganisms}

Effects of LED and UVA LED on the bacteria were compared as a function of varied exposure time (Fig. 3). The center of the petri dish was marked with " $\mathrm{X}$ " to highlight that the intensity of light source was at its maximum at the said point. From Fig. 3, it can be concluded that LED light source did not produce any observable inactivation regardless of exposure time. This pattern was observed for all studied bacteria. A summary of the inactivation efficiency of LED at various exposure time with respect to control sample is shown in Table 1. The results clearly demonstrate that LED at $460 \mathrm{~nm}$ wavelength does not possess any observable antibacterial activity irrespective of the exposure time.

Table 1 Effects of varied exposure time on bacterial inactivation.

\begin{tabular}{|c|c|c|c|c|c|c|c|c|c|c|c|c|c|c|c|}
\hline \multirow[t]{2}{*}{ Microorganism } & \multicolumn{3}{|c|}{$1 \mathrm{~min}$} & \multicolumn{3}{|c|}{$5 \min$} & \multicolumn{3}{|c|}{$15 \mathrm{~min}$} & \multicolumn{3}{|c|}{$30 \mathrm{~min}$} & \multicolumn{3}{|c|}{$60 \mathrm{~min}$} \\
\hline & c & LED & UV & C & LED & UV & C & LED & UV & C & LED & UV & c & LED & UV \\
\hline S. aureus & $\mathrm{NOI}$ & $\mathrm{NOI}$ & $\mathrm{NOI}$ & $\mathrm{NOI}$ & $\mathrm{NOI}$ & $\mathrm{NOI}$ & $\mathrm{NOI}$ & $\mathrm{NOI}$ & $\mathrm{NOI}$ & $\mathrm{NOI}$ & $\mathrm{NOI}$ & $\mathrm{OIO}$ & $\mathrm{NOI}$ & $\mathrm{NOI}$ & $\mathrm{OIO}$ \\
\hline$P$. aeruginosa & $\mathrm{NOI}$ & $\mathrm{NOI}$ & $\mathrm{NOI}$ & $\mathrm{NOI}$ & $\mathrm{NOI}$ & $\mathrm{NOI}$ & $\mathrm{NOI}$ & $\mathrm{NOI}$ & $\mathrm{NOI}$ & $\mathrm{NOI}$ & $\mathrm{NOI}$ & $\mathrm{OIO}$ & $\mathrm{NOI}$ & $\mathrm{NOI}$ & $\mathrm{OIO}$ \\
\hline E. coli & $\mathrm{NOI}$ & $\mathrm{NOI}$ & $\mathrm{NOI}$ & NOI & $\mathrm{NOI}$ & $\mathrm{NOI}$ & $\mathrm{NOI}$ & $\mathrm{NOI}$ & $\mathrm{NOI}$ & $\mathrm{NOI}$ & NOI & $\mathrm{NOI}$ & $\mathrm{NOI}$ & $\mathrm{NOI}$ & $\mathrm{OIO}$ \\
\hline
\end{tabular}

\section{Inactivation effects of UV LED on microorganisms}

Fig. 3 shows the differences in the inactivation effectiveness between UVA LED and standard LED. One striking pattern can be observed upon investigating the said figure. At time equal to $1 \mathrm{~min}$, no notable difference was observed between LED and UVA LED samples for all microorganisms. All sample petri dishes looked exactly like control sample. After $5 \mathrm{~min}$ and $15 \mathrm{~min}$ of UV light treatment, no observable antibacterial activity was noticed by the naked eye for all microorganisms. The dishes looked completely identical to control samples. Increased cloudiness was observed in $P$. aeruginosa samples at $1 \mathrm{~min}$ and $15 \mathrm{~min}$ was due to the presence of water drops.

After 30 min of treatment, the $S$. aureus sample showed inactivation activity under UVA light but treatment with standard LED did not any observable inactivation. $S$. aureus petri dish that was exposed to UVA showed inactivation properties at the center of the dish only. This is due to the fact that the UVA LED intensity was at maximum at the center of the dish hence significantly lower number of bacterial colonies were observed. However, areas closer to the edge of the dish did not demonstrate higher disinfection comparatively. This is because, only one UVA LED was used in this experiment hence the entire surface area of petri dish did not receive equal exposure intensity. Bacterial concentration increased as moved closer to the edge. This issue can be easily dealt with by combining additional UVA LEDs. P. aeruginosa sample at same exposure time showed even higher inactivation properties. Much bigger and more clearly disinfected area was seen indicating that $P$. aeruginosa, during the same exposure time showed much higher inactivation than $S$. aureus microbe. However, E. coli did not experience any inactivation even after exposure to UVA light for 30 min. E. coli petri dishes looked identical to the LED-exposed dishes, showing no antibacterial activity.

The highest inactivation was observed when the petri dishes were exposed to UVA light for $60 \mathrm{~min}$. LED-treated petri dishes, even after exposure to their respective light for $60 \mathrm{~min}$ did not produce any observable inactivation properties for all microorganisms as shown in Fig. 3. However, UVA petri dishes did produce significant bacterial inactivation for all microorganisms following $60 \mathrm{~min}$ of continuous exposure. The result suggested that standard LED light at $460 \mathrm{~nm}$ wavelength does not have any germicidal properties. Among UVA treated samples, the highest inactivation was experienced by $P$. aeruginosa, followed by $E$. coli and $S$. aureus. Although $E$. coli and $S$. aureus petri dishes were not as clearly inactivated as $P$. aeruginosa but the concentration of microorganisms at the center of the dish was significantly lower.

\section{Inhibition zone}

For comparison of inhibition zone LED and UVA LED, petri dishes were observed at all time period for all microorganisms. No inhibition zone was observed for any of the LED-treated petri dishes indicating no germicidal properties of LED at $460 \mathrm{~nm}$ wavelength. In addition, the variation of exposure time for standard LED did not have any effect on the bacterial reduction. On the other hand, UVA-treated petri dishes showed inactivation for all microorganisms. Exposure time influenced the inactivation significantly. The diameter of inhibition zone, after 60 min of exposure time, is recorded in Table 2.

Table 2 Post treatment Inhibition zone.

\begin{tabular}{lll}
\hline Microorganism & \multicolumn{2}{l}{ Inhibition Diameter $(\mathbf{m m})$} \\
\hline & LED & UVA-LED \\
S. aureus & 0 & 45 \\
P. aeruginosa & 0 & 67 \\
E. coli & 0 & 55 \\
\hline
\end{tabular}

The biggest inhibition zone diameter was observed for $P$. aeruginosa followed by $E$. coli and $S$. aureus. Results concluded that $P$. aeruginosa have higher sensitivity to $385 \mathrm{~nm}$ wavelength compared to other microorganisms. Moreover, the results also exhibited linear relationship between inactivation and exposure i.e. higher exposure time produce higher log inactivation. In a previous study (Malik et al., 2017) a comparison of standard LED and UVA LED was made for the inactivation of $\mathrm{E}$ coli. The results demonstrated that that UVA LED achieved approximately 4-log reduction as compared to 0.1-log reduction for LED treated samples.

Most existing studies carried out on UVA region mainly focused on $365 \mathrm{~nm}$ wavelength for inactivation applications (Hamamoto et al., 
2007). However, investigation of wavelengths closer to the visible spectrum have not been studied in detail. The finding of this research study clearly shows that UVA LEDs, regardless of being known as less effective, still possess germicidal activities and have higher inactivation capabilities compared to standard LED. UVA LEDs may well be used in numerous applications for inactivation of pathogenic microorganisms.

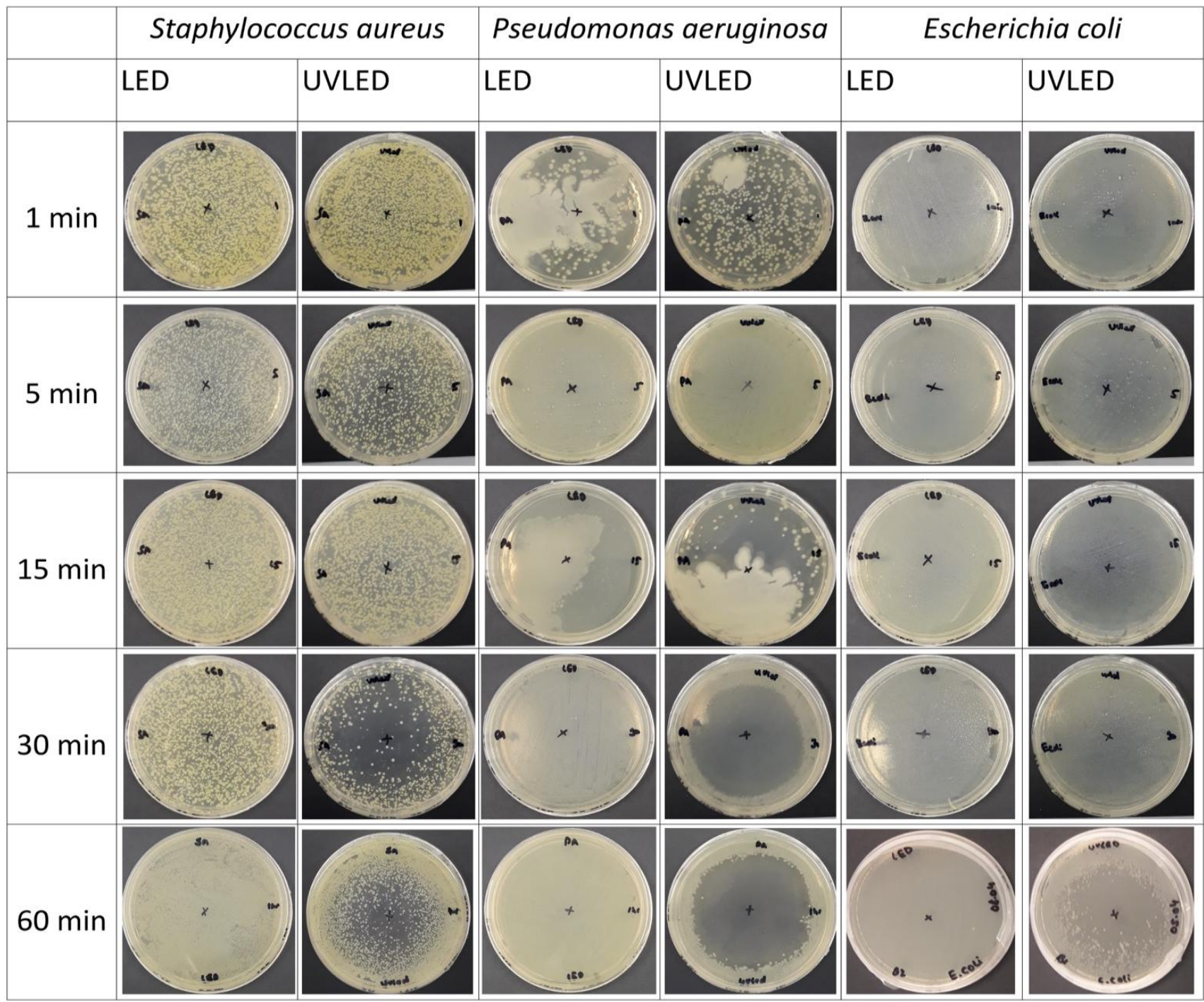

Fig. 3 Microorganism antibacterial activity as a function of varied exposure time.

\section{CONCLUSION}

Effectiveness of standard LED and UVA LED was compared for inactivation of frequently isolated pathogens in hospitals. UVA LED showed tremendous inactivation properties as compared to LED light. Inactivation efficiency was studied as a function of varied exposure time and calculation of inhibition zone was carried out to determine the disinfection effectiveness. Highest inactivation was achieved for $P$. aeruginosa. A linear relationship was witnessed between exposure time and $\log$ inactivation. As the exposure time increased so did the inactivation hence proving the importance of exposure time in achieving higher log reduction. The absence of LED's germicidal properties demonstrated that UVLED at $460 \mathrm{~nm}$ cannot to be used for disinfection applications.

\section{ACKNOWLEDGEMENT}

This research was conducted in Universiti Teknologi Malaysia (UTM). The authors gratefully acknowledge the research grant provided to this study by Research Management Centre sponsored by Ministry of Education, Malaysia. Flagship Vot: 16H19 (Research University Grant), Universiti Teknologi Malaysia, Johor Bahru, Malaysia.

\section{REFERENCES}

Al-Tawfiq, J. A., \& Tambyah, P. A. 2014. Healthcare associated infections (HAI) perspectives. Journal of Infection and Public Health, 7(4), 339344.

Anderson, D. J., Gergen, M. F., Smathers, E., Sexton, D. J., Chen, L. F., Weber, D. J., \& Rutala, W. A. 2013. Decontamination of Targeted Pathogens from Patient Rooms Using an Automated Ultraviolet-C-Emitting Device. Infection Control \& Hospital Epidemiology, 34(5), 466-471.

Aoyagi, Y., Takeuchi, M., Yoshida, K., Kurouchi, M., Yasui, N., Kamiko, N., ...Nanishi, Y. 2011. Inactivation of bacterial viruses in water using deep ultraviolet semiconductor light-emitting diode. Journal of Environmental Engineering, 137(12), 1215-1218.

Beck, S. E., Ryu, H., Boczek, L. A., Cashdollar, J. L., Jeanis, K. M., Rosenblum, J. S., ...Linden, K. G. 2017. Evaluating UV-C LED disinfection performance and investigating potential dual-wavelength synergy. Water Research, 109, 207-216.

Brannigan, E., \& Holmes, A. 2012. Healthcare associated infections-The size of the problem. In I. M. Gould \& J. W. M. van der Meer (Eds.), Antibiotic policies: Controlling hospital acquired infection (pp. 1-14). New York, NY: Springer New York.

Bukharie, H. A., Al-Zahrani, H., Rubaish, A. M., \& Abdulmohsen, M. F. 2004. Bacterial contamination of stethoscopes. Journal of Family \& Community Medicine, 11(1), 31-33. 
Chatterley, C., \& Linden, K. 2010. Demonstration and evaluation of germicidal UV-LEDs for point-of-use water disinfection. Journal of Water and Health, 8(3), 479-486.

Chen, J., Loeb, S., \& Kim, J. -H. 2017. LED revolution: Fundamentals and prospects for UV disinfection applications. Environmental Science: Water Research \& Technology, 3(2), 188-202.

Uneke, C. J. 2014. Are non-critical medical devices potential sources of infections in healthcare facilities? World Health \& Population, 15(3), 1324.

Cruickshank, M., Murphy, C. (Eds.) 2009. Reducing harm to patients from healthcare associated infections: An Australian infection prevention and control model for acute hospitals. Sydney: Australian Commission on Safety and Quality in Health Care.

Jeyakumari, D., Nagajothi, S., Kumar, P. R., Ilayaperumal, G., Vigneshwaran, S. 2016. Bacterial colonization of stethoscope used in the tertiary care teaching hospital: A potential source of nosocomial infection. International Journal of Research in Medical Sciences, 5(1), 142-145.

Dancer, S. J. 2012. Infection control 'undercover': A patient experience. Journal of Hospital Infection, 80(3), 189-191.

Donlan, R. M. 2001. Biofilms and device-associated infections. Emerging Infectious Diseases, 7(2), 277-281.

Donlan, R. M. 2008. Biofilms on central venous catheters: is eradication possible? Current Topics in Microbiology and Immunology, 322, 133161

Donlan, R. M. 2008. Biofilms on Central Venous Catheters: Is Eradication Possible? In T. Romeo (Ed.), Bacterial Biofilms (pp. 133-161). Berlin, Heidelberg: Springer Berlin Heidelberg.

Eskandarian, M. R., Choi, H., Fazli, M., \& Rasoulifard, M. H. 2016. Effect of UV-LED wavelengths on direct photolytic and $\mathrm{TiO} 2$ photocatalytic degradation of emerging contaminants in water. Chemical Engineering Journal, 300, 414-422

Grewal, H., Varshney, K., Thomas, L. C., Kok, J., \& Shetty, A. 2013. Blood pressure cuffs as a vector for transmission of multi-resistant organisms: Colonisation rates and effects of disinfection. Emergency Medicine Australasia, 25(3), 222-226.

Hamamoto, A., Mori, M., Takahashi, A., Nakano, M., Wakikawa, N., Akutagawa, M., . . . Kinouchi, Y. 2007. New water disinfection system using UVA light-emitting diodes. Journal of Applied Microbiology, 103(6), 2291-2298.

Harris, T. R., Pagan, J. G., \& Batoni, P. 2013. Optical and fluidic co-design of a UV-LED water disinfection chamber. ECS Transactions, 45(17), 11-18.

Hölz, K., Lietard, J., \& Somoza, M. M. 2017. High-power 365 nm UV LED mercury arc lamp replacement for photochemistry and chemical photolithography. ACS Sustainable Chemistry \& Engineering, 5(1), 828834.

Khurram, M., Umar, M., Akhter, T. S., Hamam-Tul-Bushra, \& Faheem, M. 2013. Frequently isolated bacteria and their culture and sensitivity pattern in a medical ICU. Journal of the College of Physicians and Surgeons Pakistan, 23(9), 681-682.

Kim, S.-J., Kim, D.-K., \& Kang, D.-H. 2016. Using UVC Light-Emitting Diodes at Wavelengths of 266 to 279 Nanometers to inactivate foodborne pathogens and pasteurize sliced cheese. Applied and Environmental Microbiology, 82(1), 11-17.

Kowalski, W. 2009. UVGI lamps and fixtures. In W. Kowalski (Ed.), Ultraviolet germicidal irradiation handbook: UVGI for air and surface disinfection (pp. 119-137). Berlin, Heidelberg: Springer Berlin Heidelberg.

Li, G. Q., Wang, W. L., Huo, Z. Y., Lu, Y., \& Hu, H. Y. 2017. Comparison of UV-LED and low pressure UV for water disinfection: Photoreactivation and dark repair of Escherichia coli. Water Research, 126, 134-143.

Linden, K. G., Thurston, J., Schaefer, R., \& Malley, J. P. 2007. Enhanced UV Inactivation of Adenoviruses under Polychromatic UV Lamps. Applied and Environmental Microbiology, 73(23), 7571-7574.

Mahida, N., Vaughan, N., \& Boswell, T. 2013. First UK evaluation of an automated ultraviolet-C room decontamination device (Tru-D TM). Journal of Hospital Infection, 84(4), 332-335.

Malik, S. A., Swee, T. T., Malek, N. A. N. N., Kadir, M. R. A., Emoto, T., Akutagawa, M., .. . Alang, T. A. I. T. 2017. Comparison of standard light- emitting diode (LED) and $385 \mathrm{~nm}$ ultraviolet A LED (UVA-LED) for disinfection of Escherichia coli. Malaysian Journal of Fundamental and Applied Sciences, 13(Special Issue on Medical Device and Technology (IMEDITECH 2017)), 430-437.

Matafonova, G., \& Batoev, V. 2018. Recent advances in application of UV light-emitting diodes for degrading organic pollutants in water through advanced oxidation processes: A review. Water Research, 132, 177-189.

McDermott, S. L., Walsh, J. E., \& Howard, R. G. 2008. A comparison of the emission characteristics of UV-LEDs and fluorescent lamps for polymerisation applications. Optics \& Laser Technology, 40(3), 487-493.

Messina, G., Burgassi, S., Messina, D., Montagnani, V., \& Cevenini, G. 2015. A new UV-LED device for automatic disinfection of stethoscope membranes. American Journal of Infection Control, 43(10), 61-66.

Messina, G., Fattorini, M., Nante, N., Rosadini, D., Serafini, A., Tani, M., \& Cevenini, G. 2016. Time effectiveness of ultraviolet C light (UVC) emitted by light emitting diodes (LEDs) in reducing stethoscope contamination. International Journal of Environmental Research and Public Health, 13(10), 940.

Nerandzic, M. M., Cadnum, J. L., Pultz, M. J., \& Donskey, C. J. 2010 Evaluation of an automated ultraviolet radiation device for decontamination of Clostridium difficile and other healthcare-associated pathogens in hospital rooms. BMC Infectious Diseases, 10(1), 197.

Oguma, K., Kita, R., \& Takizawa, S. 2016. Effects of arrangement of UV lightemitting diodes on the inactivation efficiency of microorganisms in water. Photochemistry and Photobiology, 92(2), 314-317.

Omotani, S., Tani, K., Aoe, M., Esaki, S., Nagai, K., Hatsuda, Y., . . Myotoku, M. 2018. Bactericidal effects of deep ultraviolet light-emitting diode for solutions during intravenous infusion. International Journal of Biological Sciences, 15(2), 101-107.

Shin, J. -Y., Kim, S. -J., Kim, D. -K., \& Kang, D. -H. 2016. Fundamental characteristics of deep-UV light-emitting diodes and their application to control foodborne pathogens. Applied and Environmental Microbiology, 82(1), 2-10.

Shirai, A., Watanabe, T., \& Matsuki, H. 2017. Inactivation of foodborne pathogenic and spoilage micro-organisms using ultraviolet-A light in combination with ferulic acid. Letters in Applied Microbiology, 64(2), 96102. doi:10.1111/lam.12701

Sholtes, K. A., Lowe, K., Walters, G. W., Sobsey, M. D., Linden, K. G., \& Casanova, L. M. 2016. Comparison of ultraviolet light-emitting diodes and low-pressure mercury-arc lamps for disinfection of water. Environmental Technology, 37(17), 2183-2188.

Sserwadda, I., Lukenge, M., Mwambi, B., Mboowa, G., Walusimbi, A., \& Segujja, F. 2018. Microbial contaminants isolated from items and work surfaces in the post- operative ward at Kawolo General Hospital, Uganda. BMC Infectious Diseases, 18(1), 68.

Uneke, C. J., Ndukwe, C. D., Nwakpu, K. O., Nnabu, R. C., Ugwuoru, C. D., \& Prasopa-Plaizier, N. 2014. Stethoscope disinfection campaign in a Nigerian teaching hospital: results of a before-and-after study. Journal of Infection in Developing Countries, 8 (1), 86-93.

Uneke, C. J., Ogbonna, A., Oyibo, P. G., Ekuma, U. 2008. Bacteriological assessment of stethoscopes used by medical students in Nigeria: Implications for nosocomial infection control. World health \& Population, 10(4), 53-61.

van Kleef, E., Robotham, J. V., Jit, M., Deeny, S. R., \& Edmunds, W. J. 2013. Modelling the transmission of healthcare associated infections: A systematic review. BMC Infectious Diseases, 13(1), 294.

Vilhunen, S., Särkkä, H., \& Sillanpää, M. 2009. Ultraviolet light-emitting diodes in water disinfection. Environmental Science and Pollution Research, 16(4), 439-442.

Yoshihiko, M., Masahiro, K., \& Suguru, N. 2014. Development and future of ultraviolet light-emitting diodes: UV-LED will replace the UV lamp. Semiconductor Science and Technology, 29(8), 084004.

Zachary, K. C., Bayne, P. S., Morrison, V. J., Ford, D. S., Silver, L. C., \& Hooper, D. C. 2001. Contamination of gowns, gloves, and stethoscopes with vancomycin-resistant enterococci. Infection Control \& Hospital Epidemiology, 22(9), 560-564. 